\title{
ANALYTICAL INVESTIGATION ON PAPR REDUCTION IN OFDM SYSTEMS USING GOLAY CODES
}

\author{
Sabhyata Uppal $^{*}$ - Sanjay Sharma ${ }^{* *}$ - Hardeep Singh ${ }^{* *}$
}

\begin{abstract}
Orthogonal frequency division multiplexing (OFDM) is a common technique in multi carrier communications. One of the major issues in developing OFDM is the high peak to average power ratio (PAPR). Golay sequences have been introduced to construct 16-QAM and 256-QAM (quadrature amplitude modulation) code for the orthogonal frequency division multiplexing (OFDM), reducing the peak-to-average power ratio. In this paper we have considered the use of coding to reduce the peakto-average power ratio (PAPR) for orthogonal frequency division multiplexing (OFDM) systems. By using QPSK Golay sequences, 16 and 256 QAM sequences with low PAPR are generated.
\end{abstract}

Keywords: orthogonal frequency division multiplexing (OFDM), peak to average power ratio (PAPR) golay sequences, quadrature amplitude modulation (QAM)

\section{INTRODUCTION}

Orthogonal frequency division multiplexing (OFDM) is a modulation technique which splits a high-rate bit stream into several parallel low-rate data streams and uses these substreams to modulate a number of orthogonal subcarriers by Fourier transform processing $[1,2]$. OFDM achieves robustness against multipath fading because of longer symbol duration for each subcarrier and against the intersymbol interference by inserting a guard interval in every OFDM symbol. For these reasons, OFDM has been adopted in many communication applications, such as wireless local area networks [3] and digital audio and video broadcasting [4]. In spite of many advantages, a major drawback of OFDM is that it has a high peak-to-average power ratio (PAPR). Since an OFDM signal is composed of a number of independently modulated subcarriers, it results in a high PAPR value when added up coherently. A modulation scheme with high PAPR requires a linear amplifier with a large dynamic range. The high PAPR increases the dynamic range of the analog-to-digital converter and degrades the efficiency of a linear power amplifier. Therefore, to find ways to control the PAPR of the transmitted signals in OFDM systems has become a very active research area in communication communities [5].

OFDM sequences constructed from Golay sequences not only enjoy the low PAPR values but also have better error-correcting capabilities. Since quadrature amplitude modulation signals are frequently adopted in OFDM system, the 16-QAM OFDM sequences with low PAPR are constructed from two quaternary phase-shift keying (QPSK) Golay complementary sequences in These results are extended to 256 QAM sequences .

\section{PEAK TO AVERAGE POWER RATIO}

The transmitted OFDM signal is the real part of the complex signal $S(t)=\sum_{i=0}^{n-1} c_{i}(t) e^{2 j \pi f_{i} t}$, where $f_{i}$ is the frequency of the ith carrier, $c_{i}(t)$ is constant over a symbol period of duration $\mathrm{T}$ [6]. To maintain orthogonality, the carrier frequencies are related by

$$
f_{i}=f_{0}+i \Delta f,
$$

$f_{i}=$ frequency of the ith carrier, $f_{0}=$ smallest carrier frequency, $\Delta f=$ integer multiple of the OFDM symbol rate.

Let $c_{i}(t)$ takes the value $c_{i}$ over a given symbol period, then the corresponding OFDM signal is denoted by $S_{c}(t)$ and can be expressed as

$$
S_{c}(t)=\sum_{i=0}^{n-1} c_{i} e^{2 j \pi f_{i} t} .
$$

Instantaneous envelope powe associated with the sequence $S_{c}(t)$ is given by

$$
\begin{aligned}
P_{c}(t)=\left|S_{c}(t)\right|^{2}=S_{c}(t) & S_{c}^{*}(t)= \\
& \left(\sum_{i=0}^{n-1} c_{i} e^{2 j \pi f_{i} t}\right)\left(\sum_{k=0}^{n-1} c_{k}^{*} e^{-2 j \pi f_{k} t}\right) .
\end{aligned}
$$

Letting $k=i+u, P_{c}(t)=\sum_{u=0}^{n-1} \sum_{i=0}^{n-1} c_{i} c_{i+u}^{*} e^{2 j \pi u \Delta f t}$, $P_{a}(t)=\sum_{i=0}^{n-1}\left|c_{i}\right|^{2}+\sum_{u \neq 0} \sum_{i} c_{i} c_{i+u}^{*} e^{2 j \pi u \Delta f t}$

Also the aperiodic autocorrelation of sequence ' $a$ ' of length ' $n$ ' is given by

$$
A_{c}(u)=\sum_{i=0}^{n-1} c_{i} c_{i+u}^{*}, \quad A_{c}(0)=\|c\|^{2} .
$$

\footnotetext{
* ECED, UIET, Chandigarh, Sector 25, Chandigarh. Pin 160014 India, sabhyata2@gmail.com; ** ECED, Thapar University, Patiala, Pin-147004 India, sanjay.sharma@thapar.edu, Hsingh@thapar.edu
} 
Therefore, $P_{c}(t)$ can be written as

$$
P_{c}(t)=\|c\|^{2}+\sum_{u \neq 0} A_{c}(u) e^{2 j \pi u \Delta f t},
$$

$$
\text { where }\|c\|^{2}=\sum_{i=0}^{n-1}\left|c_{i}\right|^{2} \text {. }
$$

Similarly,

$$
P_{b}(t)=\|b\|^{2}+\sum_{u \neq 0} c_{b}(u) e^{j 2 \pi u \Delta f t}
$$

Adding equation (1) \& (2), we get

$$
\begin{aligned}
& P_{a}(t)+P_{b(t)}=\|a\|^{2}+\|b\|^{2}+ \\
& \sum_{u \neq 0} c_{b}(u) e^{j 2 \pi u \Delta f t}+\sum_{u \neq 0} c_{a}(u) e^{j 2 \pi u \Delta f t} .
\end{aligned}
$$

Therefore, $P_{a}(t)+P_{b(t)}=\|a\|^{2}+\|b\|^{2}$.

\subsection{Golay Complementary Sequences}

Let us consider two sequences 'a' and 'b' of length 'n', ie $a=\left(a_{0}, a_{1}, \ldots, a_{n-1}\right), b=\left(b_{0}, b_{1}, \ldots, b_{n-1}\right), a_{i}, b_{i} \in Z_{4}$

The aperiodic autocorrelation of the sequence $c$ can be defined as

$$
A_{c}(u)=c_{u} \otimes c_{-u}^{*} .
$$

These two sequences are said to be a Golay complementary pair if sum of their aperiodic autocorrelation is a delta function [7]. Thus,

$$
C_{a}(u)+C_{b}(u)=\left(\|a\|^{2}+\|b\|^{2}\right) \delta(u)
$$

where $\delta(u)=\left\{\begin{array}{ll}1 & \text { if } u=0, \\ 0, & \text { if } u \neq 0\end{array}\right.$ is the Kronecker function.

Any sequence which is the member of the Golay complementary pair is called a Golay complementary sequence (GCS). Therefore,

$$
C_{a}(u)+C_{b}(u)= \begin{cases}0 & \text { at } u \neq 0 \\ 2 n & \text { at } u=0 .\end{cases}
$$

Since the complex envelope signal $S_{a}(t)$ and the input vector $c=\left[c_{k}\right]_{k=0}^{n-1}$ forms the Fourier series pair, their conjugates also forms a Fourier pair.

$$
\begin{gathered}
S_{c}(t) \underset{F T}{\longleftrightarrow} c_{i}, \\
S_{c}^{*}(t) \underset{F T}{\longleftrightarrow} c_{-i}^{*}, \\
S_{c}(t) S_{c}^{*}(t) \underset{F T}{\longleftrightarrow} c_{i} \otimes c_{-i}^{*} .
\end{gathered}
$$

Since $A_{c}(u)$ and $P_{c}(t)$ forms the Fourier transform pair, therefore aperiodic autocorrelation can be studied in frequency domain to obtain the knowledge about $P_{c}(t)$ in time domain [8]. Therefore

$$
P_{c}(t) \underset{F T}{\longleftrightarrow} A_{c}(u), \quad A_{a}(u)+A_{b}(u) \underset{F T}{\longleftrightarrow} P_{a}(t)+P_{b}(t)
$$

As $P_{a}(t)+P_{b}(t)=\|a\|^{2}+\|b\|^{2},\|a\|^{2}=\sum_{i=0}^{n-1}\left|a_{i}\right|^{2}=n$, $\|b\|^{2}=n$

$$
P_{a}(t)+P_{b}(t)=n+n=2 n .
$$

The PAPR of the transmitted codeword $c$ can be defined as

$$
\operatorname{PAPR}(c)=\frac{\max _{0 \leq t \leq T} P_{c}(t)}{P_{a v}} .
$$

Let $\mathrm{C}$ be the collection of all the codewords that are to be transmitted. Then the average power of the transmitted signals of $\mathrm{C}$ is

$$
P_{a v}=\sum_{c \in \mathrm{C}}\|c\|^{2} p(c)
$$

$p(c)$ is the probability of the transmission of the codeword $c$.

$$
\operatorname{PAPR}(\mathrm{C})=\max _{c \in \mathrm{C}} p(c) .
$$

Also the average power of $c$ can be expressed as

$$
\frac{1}{T} \int_{0}^{T} P_{c}(t) \mathrm{d} t=\|c\|^{2}=\sum_{k=0}^{n-1}\left|c_{k}\right|^{2}
$$

With unit energy for PSK modulation, the average power $\|c\|^{2}$ of any sequence $c$ is equal to $\sum_{k=0}^{n-1}\left|c_{k}\right|^{2}=n$.

$P_{a}(t)$ and $P_{b}(t)$ are non-negative and $P_{a}(t)+P_{b}(t)=$ $n+n=2 n$, thus we have

$$
\operatorname{PAPR}(c) \leq \frac{P_{a}(t)+P_{b}(t)}{n}=\frac{2 n}{n}=2 .
$$

\subsection{Construction of 16-QAM sequence as a sum of two QPSK sequences}

Consider $S_{Q P S K}$ be the set of QPSK constellation symbols and can be represented as

$$
S_{Q P S K}=\left\{e^{\frac{j \pi}{4}}, j e^{\frac{j \pi}{4}},-e^{\frac{j \pi}{4}},-j e^{\frac{j \pi}{4}}\right\}
$$

A 16-QAM constellation symbols can be written as the sum of two QPSK symbols. Therefore,

$$
S_{16-Q A M}=\frac{1}{\sqrt{2}} S_{Q P S K}+\sqrt{2} S_{Q P S K} .
$$




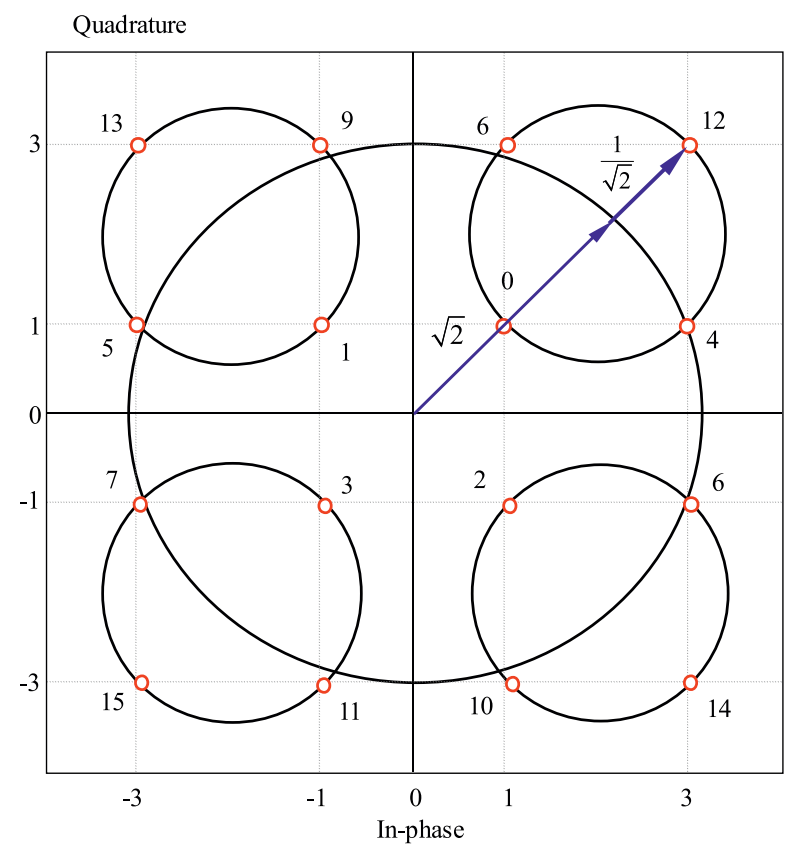

Fig. 1. 16-QAM as sum of two QPSK symbols

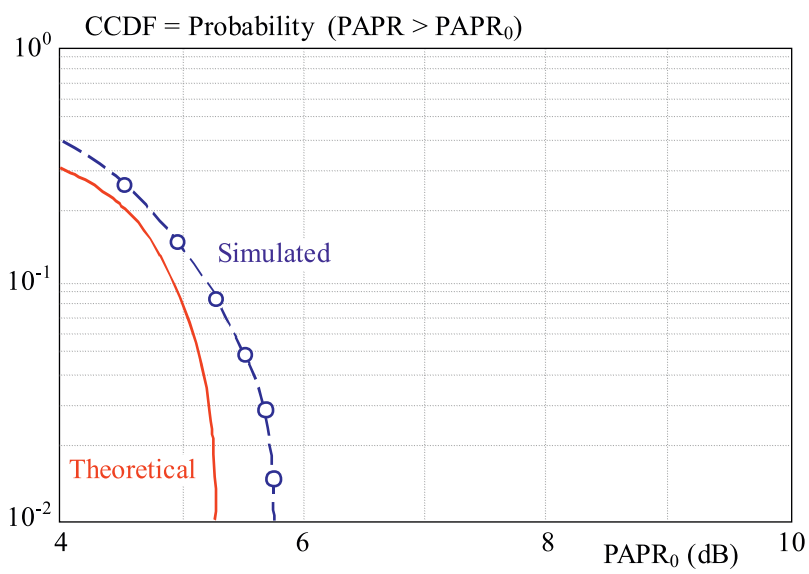

Fig. 2. PAPR for 16 QAM

Figure 1 shows the constellation diagram of 16-QAM as the sum of two QPSK symbols

Let $c=\left(c_{0}, c_{1}, \ldots, c_{n-1}\right), c_{i} \in S_{16-Q A M}$ be the 16 QAM sequence and can be associated with two QPSK sequences as

$x=\left(x_{0}, x_{1}, \ldots, x_{n-1}\right) \in Z_{4}^{n}, \quad y=\left(y_{0}, y_{1}, \ldots, y_{n-1}\right) \in Z_{4}^{n}$.

QPSK or 4-psk constellation can be realized as the set $P S K=\left\{j^{x}, x=0,1,2,3\right\}$. We can associate with any QPSK $c=\left(c_{0}, c_{1}, \ldots, c_{n-1}\right)$, a unique sequence $x_{i}=$ $\left(x_{0}, x_{1}, \ldots, x_{n-1}\right) \in Z_{4}^{n}$. Therefore, $c_{i}=j^{x_{i}}, 0 \leq i \leq$ $n-1, c_{k}=j^{x_{k}}$.

16-QAM can be expressed as

$$
16-\mathrm{QAM}=\frac{1}{\sqrt{2}} \exp \frac{j \pi}{4} P S K+\sqrt{2} \exp \frac{j \pi}{4} P S K .
$$

Substituting the values $x=0,1,2,3$ in $P S K=j^{x}$ we obtain

$$
S_{Q P S K}=\left\{e^{\frac{j \pi}{4}}, j e^{\frac{j \pi}{4}},-e^{\frac{j \pi}{4}},-j e^{\frac{j \pi}{4}}\right\}
$$

Therefore equation (6) can be written as

$$
\begin{gathered}
S_{16-Q A M}=\frac{1}{\sqrt{2}} e^{\frac{j \pi}{4}} j^{x}+\sqrt{2} e^{\frac{j \pi}{4}} j^{y}=e^{\frac{j \pi}{4}}\left(\frac{1}{\sqrt{2}} j^{x}+\sqrt{2} j^{y}\right) \\
\therefore c_{k}=e^{\frac{j \pi}{4}}\left(\frac{1}{\sqrt{2}} j^{x_{k}}+\sqrt{2} j^{y_{k}}\right), \quad 0 \leq k \leq n-1 .
\end{gathered}
$$

Assumption has been taken that the minimum Euclidean distance between signal points of 16-QAM constellation is one and the signal point is used with equal probability [9].

Average Energy

$$
c_{k}=\sum_{k=0}^{n-1}\left|c_{k}\right|^{2}=\left[\sqrt{\left(\frac{1}{2}\right)^{2}+(\sqrt{2})^{2}}\right]^{2}=2.5
$$

$\therefore$ Average power is $P_{a v}=2.5 n$.

Let $S_{x}(t)$ and $S_{y}(t)$ be the two QPSK OFDM symbols, then with $\xi=\{x, y\}$

$$
S_{\xi}(t)=\sum_{k=0}^{n-1} e^{\frac{j \pi}{4}} j^{\xi_{k}} e^{\frac{j 2 \pi k t}{T}},
$$

A 16-QAM OFDM symbol $S_{c}(t)$ can be written as the sum of the two QPSK OFDM signals. They can be represented as

$$
S_{c}(t)=S_{x}(t)+S_{y}(t)=\sum_{k=0}^{n-1} c_{k} e^{\frac{j 2 \pi k t}{T}} .
$$

Taking the value of $c_{k}$ from equation (7), we can write

$$
\begin{aligned}
S_{c}(t) & =\sum_{k=0}^{n-1}\left(\frac{1}{\sqrt{2}} j^{x_{k}}+\sqrt{2} j^{y_{k}}\right) e^{\frac{j \pi}{4}} e^{\frac{j 2 \pi k t}{T}} \\
& =\sum_{k=0}^{n-1} \frac{1}{\sqrt{2}} j^{x_{k}} e^{\frac{j \pi}{4}} e^{\frac{j 2 \pi k t}{T}}+\sum_{k=0}^{n-1} \sqrt{2} j^{y_{k}} e^{\frac{j \pi}{4}} e^{\frac{j 2 \pi k t}{T}} \\
& =\frac{1}{\sqrt{2}} \sum_{k=0}^{n-1} j^{x_{k}} e^{\frac{j \pi}{4}} e^{\frac{j 2 \pi k t}{T}}+\sqrt{2} \sum_{k=0}^{n-1} j^{y_{k}} e^{\frac{j \pi}{4}} e^{\frac{j 2 \pi k t}{T}} .
\end{aligned}
$$

From (9) and (10), we can write

$$
S_{c}(t)=\frac{1}{\sqrt{2}} S_{x}(t)+\sqrt{S}_{y}(t) .
$$

Let $x$ and $y$ be the two Golay complementary sequences of length ' $n$ '. Thus instantaneous envelope power of a 16QAM OFDM signal $S_{c}(t)$ is bounded above by

$$
\begin{gathered}
P_{c}(t)=\left|S_{c}(t)\right|^{2}=\left|\frac{1}{\sqrt{2}} S_{x}(t)+\sqrt{2} S_{y}(t)\right|^{2} \\
\leq\left|\frac{1}{\sqrt{2}} S_{x}(t)\right|^{2}\left|\sqrt{2} S_{y}(t)\right|^{2}+2 \frac{1}{\sqrt{2}}\left|S_{x}(t)\right| \sqrt{2}\left|S_{y}(t)\right| \leq \\
\left|\frac{1}{\sqrt{2}} \sqrt{2 n}\right|^{2}+|\sqrt{2} \sqrt{2 n}|^{2}+2 \frac{1}{\sqrt{2}} \sqrt{2 n} \sqrt{2} \sqrt{2 n} \leq n+4 n+4 n . \\
\therefore P_{c}(t)=9 n .
\end{gathered}
$$

As PAPR is defined in equation (5), we can write,

$$
\begin{gathered}
\text { PAPR }=\frac{\max \cdot \text { power }}{\text { avg.power }}=\frac{9 n}{2.5 n}=3.6 \\
\operatorname{PAPR}(\mathrm{db})=10 \log _{10} 3.6=5.56(\mathrm{~dB}) .
\end{gathered}
$$

Therefore PAPR for 16-QAM OFDM signal is bounded above. Figure 2.shows the comparison of theoretical and simulated results of PAPR for 16 QAM. 
292 S. Uppal - S. Sharma - H. Singh: ANALYTICAL INVESTIGATION ON PAPR REDUCTION IN OFDM SYSTEMS USING ...

\subsection{Construction of 256-QAM OFDM sequences}

256-QAM sequences can be constructed as the vector sum of four QPSK sets. Let $\mathrm{S}_{256-\mathrm{QAM}}$ denotes the 256QAM constellation symbols and can be written as the sum of four QPSK symbols [10].

$$
\begin{aligned}
& \mathrm{S}_{256-Q A M}=\frac{1}{\sqrt{2}} S_{Q P S K} \\
& \quad+\sqrt{2} S_{Q P S K}+2 \sqrt{2} S_{Q P S K}+4 \sqrt{2} S_{Q P S K} .
\end{aligned}
$$

Figure 3 shows the 256-QAM constellation diagram as the vector sum of four QPSK symbols.

Let $S_{x}(t), S_{y}(t), S_{w}(t)$ and $S_{z}(t)$ be the four QPSK OFDM signals. Consider $c=\left(c_{0}, c_{1}, \ldots, c_{n-1}\right)$, where $c_{i} \in S_{256-Q A M}$ which could be associated with four QPSK sequences.

$x=\left(x_{0}, x_{1}, \ldots, x_{n-1}\right) \in Z_{4}^{n}, y=\left(y_{0}, y_{1}, \ldots, y_{n-1}\right) \in Z_{4}^{n}$, $w=\left(w_{0}, w_{1}, \ldots, w_{n-1}\right) \in Z_{4}^{n}, z=\left(z_{0}, z_{1}, \ldots, z_{n-1}\right) \in Z_{4}^{n}$,

$$
\begin{array}{r}
c_{k}=\left(\frac{1}{\sqrt{2}} j^{x_{k}}+\sqrt{2} j^{y_{k}}+2 \sqrt{2} j^{w_{k}}+4 \sqrt{2} j\right. \\
S_{\xi}(t)=\sum_{k=0}^{n-1}\left(j^{\xi_{k}} e^{j \pi / 4} e^{j 2 \pi k t / T}\right)
\end{array}
$$

again with $\xi=\{x, y, w, z\}$.
A 256-QAM OFDM signal may be written as the weighted sum of four QPSK OFDM signals by

$$
\begin{aligned}
& S_{c}(t)=\sum_{k=0}^{n-1} c_{k} e^{j 2 \pi k t / T}= \\
& \sum_{k=0}^{n-1}\left(\frac{1}{\sqrt{2}} j^{x_{k}}+\sqrt{2} j^{y_{k}}+2 \sqrt{2} j^{w_{k}}+4 \sqrt{2} j^{z_{k}}\right) e^{j \pi / 4} e^{j 2 \pi k t / T}= \\
& \sum_{k=0}^{n-1}\left(\frac{1}{\sqrt{2}} j^{x_{k}} e^{j \pi / 4} e^{\frac{j 2 \pi k t}{T}}+\sum_{k=0}^{n-1} \sqrt{2} j^{y_{k}} e^{j \pi / 4} e^{\frac{j 2 \pi k t}{T}}+\right. \\
& \sum_{k=0}^{n-1} 2 \sqrt{2} j^{w_{k}} e^{j \pi / 4} e^{\frac{j 2 \pi k t}{T}}+\sum_{k=0}^{n-1} 4 \sqrt{2} j^{z_{k}} e^{j \pi / 4} e^{\frac{j 2 \pi k t}{T}}= \\
& \quad \frac{1}{\sqrt{2}} \sum_{k=0}^{n-1}\left(j^{x_{k}} e^{j \pi / 4} e^{\frac{j 2 \pi k t}{T}}+\sqrt{2} \sum_{k=0}^{n-1} j^{y_{k}} e^{j \pi / 4} e^{\frac{j 2 \pi k t}{T}}+\right. \\
& 2 \sqrt{2} \sum_{k=0}^{n-1} j^{w_{k}} e^{j \pi / 4} e^{\frac{j 2 \pi k t}{T}}+4 \sqrt{2} \sum_{k=0}^{n-1} j^{z_{k}} e^{j \pi / 4} e^{\frac{j 2 \pi k t}{T}}= \\
& =\frac{1}{\sqrt{2}} S_{x}(t)+\sqrt{2} S_{y}(t)+2 \sqrt{2} S_{w}(t)+4 \sqrt{2} S_{z}(t) .
\end{aligned}
$$

Instantaneous envelope power is defined by

$$
\begin{aligned}
P_{c}(t) & =\left|S_{c}(t)\right|^{2} \\
& =\left|\frac{1}{\sqrt{2}} S_{x}(t)+\sqrt{2} S_{y}(t)+2 \sqrt{2} S_{w}(t)+4 \sqrt{2} S_{z}(t)\right|^{2} \\
& \leq\left|\frac{1}{\sqrt{2}} \sqrt{2 n}+\sqrt{2} \sqrt{2 n}+4 \sqrt{2} \sqrt{2 n}\right|^{2} \\
& \leq \| \sqrt{n}+2 \sqrt{n}+4 \sqrt{n}+\left.8 \sqrt{n}\right|^{2} \leq|15 \sqrt{n}|^{2} \\
\therefore & P_{c}(t)=225 n .
\end{aligned}
$$

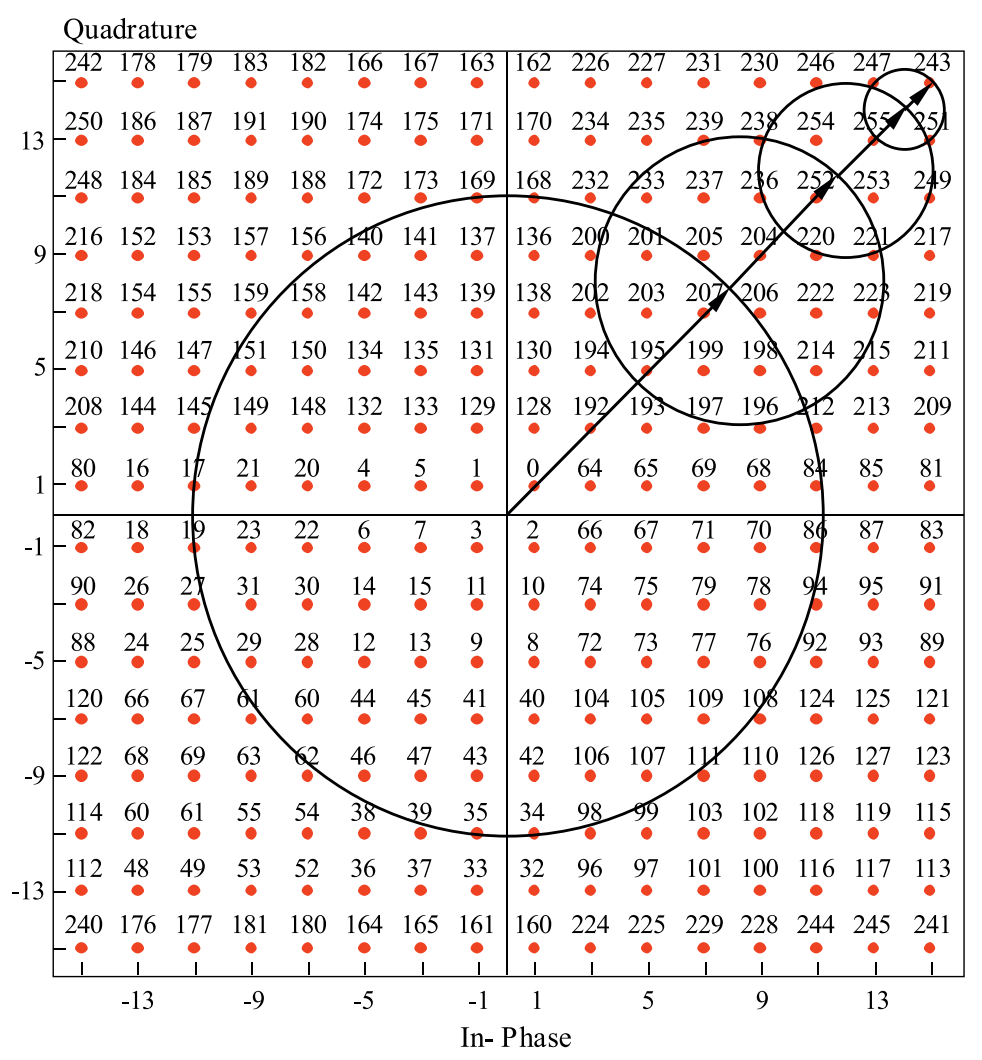

Fig. 3. 256-QAM symbols as the sum of four QPSK symbols 


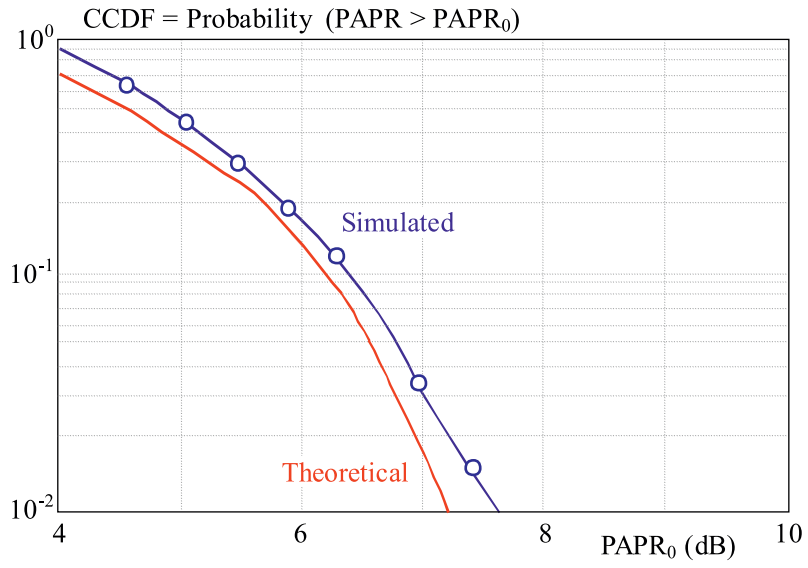

Fig. 4. PAPR for 256 QAM

Now, average energy can be calculated as

$$
\begin{aligned}
&\left\|c_{k}\right\|=\left(\sum_{k=0}^{n-1}\left|c_{k}\right|^{2}\right)^{1 / 2}= \\
& \sqrt{\left(\frac{1}{\sqrt{2}}\right)^{2}+(\sqrt{2})^{2}+(2 \sqrt{2})^{2}+(4 \sqrt{2})^{2}}=42.5 \\
& \therefore \text { Avarage power } P_{a v}=42.5 n .
\end{aligned}
$$

Therefore using the formula for PAPR, it can be calculated as

$$
\begin{aligned}
\mathrm{PAPR} & =\frac{225 n}{42,5 n}, \\
\mathrm{PAPR} & =10 \log _{10} 5.29=7.23 \mathrm{~dB}
\end{aligned}
$$

Figure 4. shows the comparison of theoretical and simulated results of PAPR for 256 QAM.

\section{CONCLUSION}

In this paper, we have proposed a technique to construct a 16-QAM and 256-QAM sequence from a combination of QPSK sequences. If these QPSK sequences are Golay sequences derived from Reed-Muller codes, then the constructed QAM sequence has a low PAPR which is suitable for OFDM systems. We have considered in this paper the reduction of peak-to average power ratio in OFDM systems for the QAM constellation, in particular for 16-QAM and 256 QAM. The methods developed in this paper can be applied to generate OFDM sequences with low PAPR for 1024-QAM constellations.

\section{REFERENCES}

[1] CIMINI, L. J. Jr.: Analysis and Simulation of a Digital Mobile Channel using Orthogonal Frequency Division Multiplexing, IEEE Trans. Commun. 33 No. 7 (July 1985), 665-675.

[2] BInghaM, J. A. C.: Multicarrier Modulation for Data Transmission: An Idea whose Time Has Comejour IEEE Commun. Mag..

[3] IEEE Std. 802.11g, "Wireless LAN Medium Access Control (MAC) and Physical Layer (PHY) Specifications," June 2003.

[4] EBU, "Digital Video Broadcasting: Framing Structure, Channel Coding, and Modulation for Digital Terrestrial Television" EN200744, V 1.2.1, Geneva, Switzerland, Jan 1999.

[5] HAN, S. H.-LEE, J. H.: An Overview of Peak-to-Average Power Ratio Reduction Techniques for Multicarricer Transmission, IEEE Wireless Commun. 12 No. 2 (Apr 2005), 56-65.

[6] DAVIS, J. A.-JEDWAB, J.: Peak-to-Mean Power Control and Error Correction for OFDM Transmission using Golay Sequences and Reed-Muller Codes, Electron. Lett. 33 No. 4 (1997), 267-268.

[7] DAVIS, J. A.-JEDWAB, J.: Peak-to-Mean Power Control in OFDM, Golay Complementary Sequences, and Reed-Muller Codes, IEEE Trans. Inf. Theory 45 No. 7 (Nov 1999), 2397-2417.

[8] GOLAY, M.: Complementary Series, IEEE Trans. Inf. Theory 7 No. 2 (Apr 1961), 82-87.

[9] RÖßING, C.-TAROKH, V.: A Construction of OFDM 16-QAM Sequences Having Low Peak Powersjour IEEE Trans. Inf. Theory.

[10] TAROKH, B.-SADJADPOUR, H. R. : Construction of OFDM M-QAM Sequences with Low Peak-to-Average Power Ratio, IEEE Trans. Commun. 51 No. 1 (Jan 2003), 25-28.

Received 12 December 2013

Sabhyata Uppal is currently working as Assistant Professor in Electronics and Communication Engineering Department of University Institute of Engineering and Technology (UIET), India. She has done her ME from Punjab Engineering College (PEC) 1999, Chandigarh. She is Pursuing PhD from Thapar University, Patiala. She has published many papers in various journals and conferences of international repute. Her main interests are Wireless System Design, Digital communication, Channel Coding etc.

Sanjay Sharma is currently working as Professor in Electronics and Communication Engineering Department of Thapar University, India. He has done his Bc Tech in ECE from REC in 1993, Jalandhar, ME in ECE from TTTI, Chandigarh in 2001 and PhD from PTU, Jalandhar in 2006. He has completed all his education with honours. He has published many papers in various journals and conferences of international repute. He has to his credit the implementation of research projects worth 12000 USD. His main interests are VLSI Signal Processing, Wireless System Design using Reconfigurable Hardware, Digital communication, Channel Coding etc.

Hardeep Singh, Currently working as Assistant Professor, Department of Electronics \& Communication Engineering, Thapar University, Patiala-147004, Punjab. His research areas are Optical Communication, Fiber, Non-linearities and dispersion, Optical Networks, Electronic Communication Circuits, Semiconductor Devices and Analog Electronic Circuits. 